\title{
A study on the quality of life of patients with type 2 diabetes mellitus and hypertension presenting to a tertiary care hospital in Southern India
}

Sir,

Chronic diseases such as diabetes and hypertension need lifelong treatment and daily self-management. Most health interventions aim to improve the quality of life of persons affected by disease. ${ }^{[1]}$ The quality of life is a vital component in disease management, because a poor quality of life leads to diminished self-care that in turn leads to worsened disease control, increased risks for complications, and exacerbation of disease. ${ }^{[2]}$ Higher burden of the disease and complications would in turn increase hospitalization and contribute to higher health care costs. Thus, it is apparent that the quality of life issues are imperative and predict how well an individual would be able to handle his disease and maintain his long-term health and well-being. ${ }^{[2]}$ Health-related quality of life (HRQoL) could be improved by early detection of the disease, adequate control of blood pressure/blood glucose levels, and patient education and empowerment. ${ }^{[1]}$ The present study was thus designed to assess the effect of chronic diseases [type-2 diabetes mellitus (DM) and/ or hypertension] on the quality of life.

It was a cross-sectional study that was carried out over a period of two months in a tertiary care hospital in Southern India. Institutional ethical clearance was obtained before the initiation of the study (IEC62/2011). The study included both male and female patients $\geq 20$ years of age, diagnosed with hypertension and/or type $2 \mathrm{DM}$ and on treatment for at least 1-year duration, attending the outpatient clinic of the medicine department. Patients with existing comorbidities such as stroke, cardiac or renal diseases, pregnant females, admitted inpatients, and patients referred only for consultation to the outpatient department (OPD) were excluded. The questionnaire was prepared using the World Health Organization Quality of Life-brief (WHOQOL-BREF) questionnaire ${ }^{[3]}$ and diabetes quality of life brief clinical inventory. ${ }^{[4]}$

Of the 52 patients, 40 patients had diabetes alone, 10 patients had only hypertension, while 2 patients had both diabetes and hypertension. In the study $40 \%$ of the patients belonged to the age group of $40-49$ years. Males constituted $69 \%$ of the study subjects. The mean duration of the illness in diabetics and the hypertensive was 7.8 years and 4.2 years, respectively. In the study, body mass index was in the normal range among $41(78.8 \%)$ patients. Around nine $(17 \%)$ patients had a body mass index of $>25$. The 
quality of life was average for $24(46.2 \%)$ subjects and poor for $15(28.8 \%)$ patients. Out of the 17 patients who were in a state of therapeutic control, eight $(47.1 \%)$ patients had a good quality of life, while only two (11.7\%) had a poor quality of life. Out of the 25 patients whose diabetes was not controlled, only two (8\%) patients had a good quality of life; while 12 (48\%) patients had a poor quality of life. Thus, the state of therapeutic control was found to be statistically associated (chi square $=11.14, P$-value $<0.05$ ) with the quality of life of a patient. Similarly, out of the nine hypertensive patients who had their blood pressure under control, three (33\%) patients had a good quality of life; while out of the three patients whose blood pressure was not controlled, none of the patients had a good quality of life. But these results were not found to be statistically significant.

The study identified that elderly people more than 60 years of age had a good quality of life, in contrast to patients in the age group of 40-49 years, who were found to have a poor quality of life, as depicted in Table 1 . Across all categories of HRQoL, the proportion of males was more than females. Table 2 shows that, the domain "physical functioning" was most severely affected as most of the patients were found to have a poor quality of life in this domain, in the present study, while a study in Croatia ${ }^{[1]}$ reported that all dimensions of HRQoL were poorer in people with diabetes and hypertension compared with healthy individuals. In contrast, the scores for both the diabetic cases and controls were reported to be low in all the domains, such as physical health, psychological health, and environmental domains by Jain et al. from central India. ${ }^{[2]}$ According to the WHOQOL-BREF questionnaire, the response of $60-70 \%$ of patients was average to good for the overall and general health-related questions. But for the physical, psychological, social, and environmental domains, majority of the patients responded as poor to good in a study among the hypertensives from Gujarat, India. ${ }^{[5]}$

Chronic diseases such as diabetes and hypertension affect the quality of life of the patient and thus interfere with the day-to-day activities. In the present study, the domain of "physical functioning" was found to be most severely affected due to chronic illness. Therefore, health care providers need to understand the physical, emotional, and social impact of these chronic diseases. The study addtionally shows a significant association between the "state of therapeutic control" and the "quality of life" of patients with diabetes. Such patient-centered knowledge can be incorporated into chronic disease management strategies designed to improve or enhance function in everyday life and improve or enhance health-related quality of life. As therapeutic control contributes significantly

\begin{tabular}{lccc}
\hline \multirow{2}{*}{ Table 1: Demographic factors affecting quality of life } \\
\hline Variables & \multicolumn{3}{c}{ Quality of life categories } \\
\cline { 2 - 4 } & Good $\boldsymbol{n}(\%)$ & Average $\boldsymbol{n}(\%)$ & Poor $\boldsymbol{n}(\%)$ \\
\hline Age group (years) & 0 & $1(4.2)$ & $2(13.3)$ \\
$20-29$ & $1(7.7)$ & $3(12.5)$ & $1(6.7)$ \\
$30-39$ & $4(30.8)$ & $11(45.8)$ & $6(40.0)$ \\
$40-49$ & $2(15.4)$ & $2(8.3)$ & $3(20.0)$ \\
$50-59$ & $6(46.2)$ & $7(29.2)$ & $3(20.0)$ \\
$>60$ & $11(84.6)$ & $16(66.7)$ & $09(60.0)$ \\
Gender & $02(15.4)$ & $08(33.3)$ & $06(40.0)$ \\
Male &
\end{tabular}

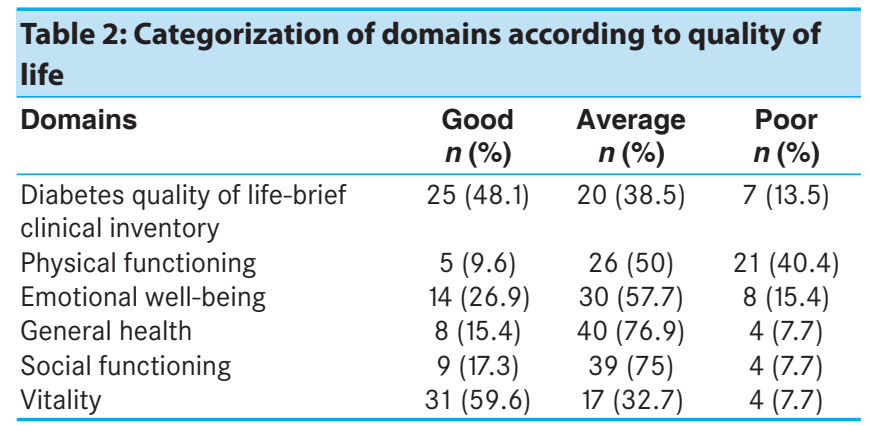

to the quality of life of patients with diabetes and/or hypertension, measures toward the maintenance of a longterm state of therapeutic control should be the primary aim of disease management.

Financial support and sponsorship

The study was funded by Indian Council of Medical Research - Short Term Studentship program - STS Ref ID: $2011-01247$.

\section{Conflicts of interest}

There are no conflicts of interest.

Varun Gosain, Chythra R Rao ${ }^{1}$

Department of General Medicine, Adichunchanagiri Institute of Medical Sciences, Balagangadharanatha Nagara, Nagamangala Taluk, Mandya District, Karnataka, ${ }^{1}$ Department of Community Medicine, Kasturba Medical College, Manipal University, Manipal, Karnataka, India

Corresponding Author: Dr. Chythra R Rao, Department of Community Medicine, Kasturba Medical College, Manipal University, Manipal - 576 104, Karnataka, India. E-mail: chythra.raj@manipal.edu

\section{REFERENCES}

1. Poljicanin T, Ajduković D, Sekerija M, Pibernik-Okanović M, Metelko Z, Vuletić Mavrinac G. Diabetes mellitus and hypertension have comparable adverse effects on health-related quality of life. BMC Public Health 2010;10:12. 
2. Jain V, Shivkumar S, Gupta O. Health-related quality of life (hr-qol) in patients with type 2 diabetes mellitus. N Am J Med Sci 2014;6:96-101.

3. WHO quality of life brief questionnaire. Available from: http://www. who.int/substance_abuse/research_tools/en/english_whoqol.pdf. [Last accessed on 2011 Feb 01].

4. Burroughs ET, Desikan R, Waterman MB, Gilin D, McGill J. Development and validation of the diabetes quality of life brief clinical inventory. Diabetes Spectrum 2004;17:41-9.

5. Oza BB, Patel BM, Malhotra SD, Patel VJ. Health related quality of life in hypertensive patients in a tertiary care teaching hospital. J Assoc Physicians India 2014;62:22-9.

How to cite this article: Gosain V, Rao CR. A study on the quality of life of patients with type 2 diabetes mellitus and hypertension presenting to a tertiary care hospital in Southern India. J Soc Health Diabetes 2016;4:45-7.

\begin{tabular}{|l|l|}
\hline \multicolumn{2}{|c|}{ Access this article online } \\
\hline Quick Response Code: & Website: \\
\hline & www.joshd.net \\
\hline & \\
\hline
\end{tabular}

This is an open access article distributed under the terms of the Creative Commons Attribution-NonCommercial-ShareAlike 3.0 License, which allows others to remix, tweak, and build upon the work non-commercially, as long as the author is credited and the new creations are licensed under the identical terms. 